\title{
Efficacy and Safety of the Combination of Nano- Liposomal Irinotecan and 5-Fluorouracil/L- Leucovorin in Unresectable Advanced Pancreatic Cancer: A Real-World Study
}

Hidetoshi Yasuoka

Takasaki General Medical Center

Atsushi Naganuma

Takasaki General Medical Center

Eishin Kurihara

Ota Memorial Hospital: Ota Kinen Byoin

Tsutomu Kobatake

Ota Memorial Hospital: Ota Kinen Byoin

Masashi ljima

Ota Memorial Hospital: Ota Kinen Byoin

Yuki Tamura

Buffalo General Medical Center

Yuhei Suzuki

Takasaki General Medical Center

Takashi Hoshino

Takasaki General Medical Center

Hisashi Hosaka

Gunma Prefectural Cancer Center: Gunma Kenritsu Gan Center

Takeshi Hatanaka

Gunma Saiseikai Maebashi Hospital

Sachiko Yoshida

Gunma Saiseikai Maebashi Hospital

Ryusuke Aihara

Gunma Saiseikai Maebashi Hospital

Yasuo Hosouchi

Gunma Saiseikai Maebashi Hospital

Norihiro Ishii

Gunma Daigaku Daigakuin Igakukei Kenkyuka Igakubu

Kenichiro Araki

Gunma Daigaku Daigakuin Igakukei Kenkyuka Igakubu 


\section{Ken Shirabe}

Gunma Daigaku Daigakuin Igakukei Kenkyuka Igakubu

Satoru Kakizaki ( $\square$ kakizaki@gunma-u.ac.jp )

Takasaki General Medical Center https://orcid.org/0000-0003-0224-7093

\section{Research Article}

Keywords: nano-liposomal irinotecan (nal-IRI), unresectable advanced pancreatic cancer; second-line treatment, real world data

Posted Date: January 12th, 2022

DOI: https://doi.org/10.21203/rs.3.rs-1233620/v1

License: (c) (i) This work is licensed under a Creative Commons Attribution 4.0 International License.

Read Full License 


\section{Abstract}

Aim: This retrospective study investigated the efficacy and safety of nano-liposomal irinotecan (nal-IRI) plus 5-fluorouracil/I-leucovorin (5-FU/I-LV) treatment in the second-line or later setting for advanced pancreatic cancer under real-world conditions.

Methods: Between June 2020 and September 2021, a total of 44 patients with unresectable advanced pancreatic cancer treated with nal-IRI + 5-FU/I-LV in our affiliated hospitals were included. The prognosis, predictive factors (including systemic inflammation-based prognostic indicators), and adverse events were investigated.

Results: The median age was 68 (interquartile range [IQR] 62-73) years old, and 22 patients (50.0\%) were male. Concerning tumor factors, 9 patients (20.5\%) had local advanced disease, and 35 patients (79.5\%) had metastases. Twenty-five of the 44 patients were receiving second-line treatment, and 19 were receiving third-line or later treatment. The median overall survival (OS) and progression free survival (PFS) were 9.0 (range, 0.7-15.4) months and 4.4 (range, 0.6-15.4) months, respectively. The overall response rate (ORR) was $5.3 \%$. The disease control rate (DCR) was $44.7 \%$. Patients with a neutrophil-tolymphocyte ratio (NLR) of $>2.7$ had a significant risk of a poor $\mathrm{OS}(\mathrm{HR}=0.275, \mathrm{P}=0.017)$. Adverse events were manageable, although gastrointestinal symptoms and neutropenia were observed. The most common grade $\geq 3$ adverse event was neutropenia, which was reported in $20 \%$ of patients.

Conclusions: Nal-IRI + 5-FU/I-LV therapy was considered to be a useful regimen as second-line or later treatment for unresectable advanced pancreatic cancer, even in clinical practice.

\section{Brief Abstract}

Nano-liposomal irinotecan (nal-IRI) plus 5-fluorouracil/I-leucovorin (5-FU/I-LV) treatment was considered to be a useful regimen as second-line or later treatment for unresectable advanced pancreatic cancer, even in clinical practice.

\section{Introduction}

Despite recent advances in diagnostic technology and anticancer drugs, pancreatic cancer continues to have a poor prognosis worldwide [1]. It is the fourth leading cause of cancer-related death in the United States and Japan $[1,2]$. Approximately $70 \%-90 \%$ of patients with pancreatic cancer are diagnosed at an advanced stage, and the 5-year overall survival (OS) rate is only $8 \%-11 \%[3-5]$.

As first-line treatment for advanced pancreatic cancer, combination therapy with gemcitabine plus nabpaclitaxel (GEM + Nab-PTX) or folinic acid, 5-fluorouracil (5-FU), irinotecan and oxaliplatin (FOLFIRINOX) has shown a survival benefit compared to gemcitabine monotherapy [6, 7]. However, the OS with first-line treatment remains less than one year on average $[6,7]$. Similarly, the progression-free survival (PFS) for the recommended first-line regimens, such as gemcitabine, GEM + Nab-PTX, and FOLFIRINOX, are only 
3.4, 5.5, and 6.4 months, respectively [6-8]. As a result, most patients require second-line or later regimens.

Nano-liposomal irinotecan (nal-IRI) consists of pegylated liposomes containing irinotecan sucrosofate salt, a topoisomerase I inhibitor $[9,10]$. Liposomal encapsulation reduces premature liver metabolism and conversion of irinotecan to the highly active SN-38 metabolite $[9,10]$. Nal-IRI exhibits a lower maximum concentration of free irinotecan in plasma, a longer half-life and a greater area under the curve in plasma for SN-38 $[9,10]$ than non-liposomal irinotecan $[9,10]$. This prolongs tumor exposure to SN-38 above its antitumor activity threshold and increases the SN-38 levels in tumor tissue compared with plasma $[9,10]$.

The phase III NAPOLI-1 trial demonstrated a better median OS with nal-IRI + 5-FU/I-leucovorin (5-FU/I-LV) than with 5-FU/I-LV (6.1 months vs. 4.2 months) [11, 12]. A phase II trial in Japan also demonstrated a similar median OS of 6.3 months for nal-IRI + 5-FU/I-LV with a tolerable safety profile [13]. In Japan, this combination therapy has been available since June 2020 in the real-world setting. Therefore, there is limited post-approval real-world data regarding its efficacy, safety and optimal sequencing in Japan. The NAPOLI-1 trial only enrolled patients who failed prior gemcitabine-based therapy.

Given the above, the present study investigated the efficacy and safety of nal-IRI + 5-FU/I-LV treatment under real-world conditions in Japanese patients. The predictive factors, including systemic inflammation-based prognostic indicators, were investigated.

\section{Methods}

\section{Patients}

Between June 2020 and September 2021, a total of 44 Japanese patients with advanced pancreatic cancer receiving nal-IRI + 5-FU/I-LV treatment at Takasaki General Medical Center and its affiliated hospitals were included, and none were excluded from the current retrospective study. Patients were diagnosed with pancreatic cancer based on typical radiological findings or pathological findings. The authors retrospectively examined the medical records, collected patient characteristics and analyzed the outcomes, including the tumor response, OS, PFS and adverse events (AEs).

This study protocol was approved by the ethics committee of each institution and was conducted in compliance with the 1975 Declaration of Helsinki.

\section{nal-IRI + 5-FU/I-LV treatment and the assessment of the tumor response and AEs}

Nal-IRI $\left(80 \mathrm{mg} / \mathrm{m}^{2}\right)$ was administered by intravenous infusion over $90 \pm 10$ minutes, followed by 200 $\mathrm{mg} / \mathrm{m}^{2} \mathrm{I}$-LV via intravenous infusion over $2 \mathrm{~h}$ and then $2400 \mathrm{mg} / \mathrm{m}^{2} 5-\mathrm{FU}$ via intravenous infusion over $46 \pm 3 \mathrm{~h}$, every 2 weeks. During screening, patients were tested for the presence of uridine diphosphate glucuronosyltransferase 1 A 1 (UGT1A1)*28 and UGT1A1*6 alleles to determine the starting dose for nal- 
IRI. A patient found to be homozygous with UGT1A $1 * 28$ or UGT1A $1 * 6$ or double heterozygous received a reduced starting dose of nal-IRI $\left(60 \mathrm{mg} / \mathrm{m}^{2}\right)$. Treatment continued until the appearance of disease progression or unacceptable AEs.

Contrast-enhanced computed tomography (CT) or magnetic resonance imaging was carried out every 4-8 weeks. The tumor response was evaluated by Response Evaluation Criteria in Solid Tumors (RECIST) version 1.1. The overall response rate (ORR) was defined as the sum of the complete response (CR), and partial response (PR). The disease control rate (DCR) was defined as the sum of the CR, PR and stable disease (SD) rates. The OS was defined as the period from the day of initial nal-IRI + 5-FU/I-LV treatment to the day of death or last visit. The PFS was defined as the period from the day of initial nal-IRI + 5-FU/ILV treatment to the day of the presence of disease progression or death.

The performance status was evaluated by the Eastern Cooperative Oncology Group [14]. AEs related to nal-IRI + 5-FU/I-LV treatment were assessed by the Common Terminology Criteria for Adverse Events version 5.0 [15]. The serum levels of CEA and CA19-9 were measured at baseline. The modified Glasgow Prognostic Score (mGPS) was determined as described previously $[16,17]$. Patients were stratified into 3 mGPS groups: mGPS 0 (CRP $<0.5$ and albumin $>3.5 \mathrm{~g} / \mathrm{dL}$ ), mGPS 1 (CRP $>0.5 \mathrm{mg} / \mathrm{L}$ or albumin $<3.5$ $\mathrm{g} / \mathrm{dL}$ ), and $\mathrm{mGPS} 2$ (CRP $>0.5 \mathrm{mg} / \mathrm{L}$ and albumin $<3.5 \mathrm{~g} / \mathrm{dL}$ ). The neutrophil-to-lymphocyte ratio (NLR) [18], platelet-to-lymphocyte ratio (PLR) and CRP/Alb ratio were calculated. The prognostic nutritional index $(\mathrm{PNI})$ was calculated as follows: $\mathrm{PNI}=[10 \times$ serum albumin $(\mathrm{g} / \mathrm{dL})]+[0.005 \times$ total lymphocyte count $\left.\left(/ \mathrm{mm}^{3}\right)\right]$.

\section{Statistical analyses}

Categorical variables are presented as numbers and percentages, and continuous variables are presented as the median (interquartile range [IQR]). Differences between groups were analyzed by Fisher's exact probability test and the Mann Whitney $U$ test when a significant difference was obtained by the KruskalWallis test. The prognosis was assessed using a Cox hazard analysis, the Kaplan-Meier method and a log-rank test. All statistical analyses were performed using the IBM SPSS (Statistics Package for Social Sciences) Statistics 25 software program (Chicago, IL, USA). P values of $<0.05$ were considered to indicate statistical significance.

\section{Results}

Patient characteristics are summarized in Table 1. The median age of all patients was 68 (IQR, 62-73) years old, and there were $22(50.0 \%)$ men. The median body mass index (BMI) before nal-IRI + 5-FU/I-LV treatment was 21.1 (IQR: 19.4-23.8) kg/m². The median BMI before first-line treatment was 21.5 (IQR: 19.4-25.1) kg/m². The body weight ratio (before nal-IRI + 5-FU/I-LV / before first-line treatment) was 98.1\% (IQR: $91.3 \%-101.5 \%)$. Concerning tumor factors, 9 patients (20.5\%) had local advanced disease, and 35 patients (79.5\%) had metastases. The PS was 0, 1 and 2 in 17 (38.6\%), 24 (54.6\%) and 3 patients (6.8\%), respectively. The serum levels of CEA and CA19-9 at baseline were 9.4 (IQR, 3.9-48.4) $\mathrm{ng} / \mathrm{ml}$ and 1585 (IQR, 121-6264) U/ml, respectively. A history of first-line treatment with GEM + Nab-PTX, 
mFOLFIRINOX and S-1 was noted in 37 (84.1\%), 2 (4.5\%) and 5 patients (11.4\%), respectively. Twenty-five (56.8\%), 13 (29.5\%), 4 (9.1\%), 1 (2.3\%), 1 patient (2.3\%) received nal-IRI + 5-FU/I-LV treatment as 2nd-, 3rd-, 4th-, 5th- and 6th-line treatment, respectively. Sixteen (38.1\%) of the 42 tested patients were positive for UGT1A1 polymorphism. Among these 16 patients, 5 needed to have the starting dose of nal-IRI reduced to $60 \mathrm{mg} / \mathrm{m}^{2}$. These five patients included one patient with UGT1A1*6/*6, two with UGT1A $1 * 28 / * 28$ and one with UGT $1 \mathrm{~A} 1 * 6 / * 28$ 
Table 1

Patients' baseline characteristics.

\section{Characteristics}

Patients, n (\%)

Sex, n (\%)

Age, years (IQR)

BMI, $\mathrm{kg} / \mathrm{m}^{2}(\mathrm{IQR})$

Cancer stage, $\mathrm{n}(\%)$

Alb, g/dL (IQR)

CRP (IQR)

mGPS (\%)

NLR (IQR)

PLR (IQR)

CRP/Alb ratio (IQR)

PNI (IQR)

CEA (IQR)

CA19-9 (IQR)

Line of therapy at which nal-IRI + 5-FU/I-LV was administered (\%)
Number (\%) or median (IQR)

44 (100)

Male

$22(50.0)$

Female

$22(50.0)$

68 (62-73)

$21.1(19.4-23.8)$

local/locally

advanced

metastatic

35 (79.5)

$0 / 1 / 2$

17 (38.6)/ $24(54.6) / 3$

(6.8)

$3.6(3.3-3.8)$

$0.33(0.14-0.88)$

$0 / 1 / 2$

18 (40.9)/ 18 (40.9)/ 8 (18.2)

3.45 (2.64-5.08)

182.8 (141.7-247.8)

$0.09(0.03-0.28)$

41.7 (38.7-44.6)

$9.4(3.9-48.4)$

1585 (121-6264)

25 (56.8)

13 (29.5)

4 (9.1)

1 (2.3)

6th

1 (2.3)

IQR, interquartile range; BMI, body mass index; Alb, albumin; mGPS, modified Glasgow Prognostic Score; NLR, neutrophil-to-lymphocyte ratio; PLR, platelet-to-lymphocyte ratio; PNI, prognostic nutritional index; GEM, gemcitabine; Nab-PTX, nab-paclitaxel, mFOLFIRINOX, modified FOLFIRINOX; UGT, uridine diphosphate glucuronosyltransferase. 


\begin{tabular}{|c|c|c|}
\hline Characteristics & & $\begin{array}{l}\text { Number (\%) or median } \\
\text { (IQR) }\end{array}$ \\
\hline \multirow[t]{3}{*}{ First-line cancer therapy (\%) } & GEM + Nab-PTX & $37(84.1)$ \\
\hline & mFOLFIRINOX & $2(4.5)$ \\
\hline & $S-1$ & $5(11.4)$ \\
\hline \multirow[t]{3}{*}{ UGT1A1 status (\%) } & $\begin{array}{l}\text { Polymorphism } \\
\text { present }\end{array}$ & $16(36.4)$ \\
\hline & No polymorphism & $26(59.1)$ \\
\hline & Not tested & $2(4.5)$ \\
\hline \multicolumn{3}{|c|}{$\begin{array}{l}\text { IQR, interquartile range; BMI, body mass index; Alb, albumin; mGPS, modified Glasgow Prognostic } \\
\text { Score; NLR, neutrophil-to-lymphocyte ratio; PLR, platelet-to-lymphocyte ratio; PNI, prognostic } \\
\text { nutritional index; GEM, gemcitabine; Nab-PTX, nab-paclitaxel, mFOLFIRINOX, modified FOLFIRINOX; } \\
\text { UGT, uridine diphosphate glucuronosyltransferase. }\end{array}$} \\
\hline
\end{tabular}

\section{The OS and PFS}

In the analysis of the OS, events occurred in 26 patients (59.1\%), with a median follow-up period of 7.2 months (95\% confidence interval [Cl] 6.0-8.4 months). The Kaplan-Meier curve showed that the median OS of all patients was 9.0 months (95\% Cl 7.10-10.91 months; Figure 1a). Figure 2 shows the KaplanMeier curve of the OS according to the systemic inflammation-based prognostic indicators. The patients with an NLR $<2.7$ (median, not reached) had a significantly better survival than those with an NLR $\geq 2.7$ (median 6.0 months; $95 \% \mathrm{Cl} 4.98-6.95$ months; $\mathrm{P}=0.002$; Figure $2 \mathrm{~b}$ ). The patients with a CRP/Alb ratio < 0.3 (median 9.8 months; $95 \% \mathrm{Cl} 7.82-11.71$ ) also had a significantly better survival than those with a CRP/Alb ratio $\geq 0.3$ (median 5.1 months; $95 \% \mathrm{Cl}$ 0.0-10.31 months; $\mathrm{P}=0.017$; Figure $2 \mathrm{~d}$ ). There were no significant differences in the OS regarding the mGPS score, PLR or PNI. The OS in the patients treated with nal-IRI + 5-FU/I-LV as 2nd-line treatment (median 9.0 months; $95 \% \mathrm{Cl}$ 6.2-11.8 months) was not significantly different from that in those treated with nal-IRI + 5-FU/I-LV as 3rd-line or later treatment (median 8.2 months; $95 \% \mathrm{Cl}$ 2.5-14.0 months; $\mathrm{P}=0.802$ ).

Figure 3 shows the receiver operator characteristic (ROC) analyses for the OS at the six-month follow-up. The NLR (Figure $3 b, P=0.019$ ) and CRP/Alb ratio (Figure 3d, $P=0.028$ ) were significantly associated with the OS at the 6-month follow-up, whereas the mGPS, PLR and PNI did not influence the OS at the 6-month follow-up. The area under the curve (AUC) of variables was as follows: mGPS, 0.656 (95\% Cl 0.478-0.835, $\mathrm{P}=0.086$ ): NLR, 0.726 (95\% Cl 0.578-0.876, $\mathrm{P}=0.019)$ : PLR, 0.568 (95\% Cl 0.391-0.745, $\mathrm{P}=0.45): \mathrm{CRP} / \mathrm{Alb}$ ratio, 0.701 (95\% Cl 0.521-0.881, $\mathrm{P}=0.028)$ : $\mathrm{PNI}, 0.600$ (95\% $\mathrm{Cl} 0.399-0.802, \mathrm{P}=0.327)$.

Figure $1 \mathrm{~b}$ shows the PFS of all patients. Events were observed in 36 patients $(81.8 \%)$ in the analysis of the PFS. The median PFS in all patients was estimated to be $4.4(95 \% \mathrm{Cl} 2.4-6.4)$ months. The PFS in the patients treated with nal-IRI + 5-FU/I-LV as 2nd-line treatment (median 4.4 months; $95 \%$ Cl 2.1-6.8 
months) was not significantly different from that in those treated with nal-IRI + 5-FU/I-LV as 3rd-line or later treatment (median 4.4 months; $95 \% \mathrm{Cl}$ 0.8-8.0 months; $\mathrm{P}=0.583$ ).

\section{The ORR and DCR}

The results associated with the tumor response are shown in Table 2. According to the RECIST, 2 patients (4.5\%) had PR, 15 (34.1\%) had SD, 21 (47.8\%) had PD, and 6 patients (13.6\%) were not evaluable (NE), while no patients had CR. Thus, the ORR and DCR in all patients were calculated to be $5.3 \%(2 / 38)$ and $44.7 \%(17 / 38)$, respectively.

Table 2

The overall response and disease control rates.

\begin{tabular}{|ll|}
\hline & All patients $(\mathrm{n}=44)$ \\
\hline Complete response, $\mathrm{n}(\%)$ & $0(0)$ \\
\hline Partial response, $\mathrm{n}(\%)$ & $2(4.5)$ \\
\hline Stable disease, $\mathrm{n}(\%)$ & $15(34.1)$ \\
\hline Progressive disease, $\mathrm{n}(\%)$ & $21(47.8)$ \\
\hline Not evaluable, $\mathrm{n}(\%)$ & $6(13.6)$ \\
\hline Overall response rate (\%) & $2 / 38(5.3 \%)$ \\
\hline Disease control rate (\%) & $17 / 38(44.7 \%)$ \\
\hline
\end{tabular}

\section{Factors predicting the OS according to univariate and multivariate analyses}

Table 3 shows predictive factors associated with the OS in patients treated with nal-IRI + 5-FU/I-LV by univariate and multivariate analyses. According to univariate analyses, an NLR $\geq 2.7(P=0.004)$ and $\mathrm{CRP} / \mathrm{Alb}$ ratio $\geq 0.3(\mathrm{P}=0.023)$ were significant predictive factors of the OS. According to multivariate analyses, only an NLR $\geq 2.7$ was selected as a significant predictive factor of the OS $(P=0.017)$. 
Table 3

Factors predicting the overall survival in patients treated with nal-IRI $+5-\mathrm{FU} / \mathrm{I}-\mathrm{LV}$ by univariate and multivariate analyses.

\begin{tabular}{|c|c|c|c|c|c|c|c|}
\hline \multirow[t]{2}{*}{ Variables } & & \multicolumn{3}{|c|}{ univariate analyses } & \multicolumn{3}{|c|}{ multivariate analyses } \\
\hline & & $\begin{array}{l}\text { Hazard } \\
\text { ratio }\end{array}$ & $95 \% \mathrm{Cl}$ & $P$-value & $\begin{array}{l}\text { Hazard } \\
\text { ratio }\end{array}$ & $95 \% \mathrm{Cl}$ & $\begin{array}{l}P \\
\text { value }\end{array}$ \\
\hline \multirow[t]{2}{*}{ Sex, n (\%) } & Male & 0.985 & $\begin{array}{l}0.454- \\
2.136\end{array}$ & 0.969 & 0.677 & $\begin{array}{l}0.282- \\
1.622\end{array}$ & 0.38 \\
\hline & Female & 1 & & & 1 & & \\
\hline \multirow[t]{2}{*}{ Age, years } & $<70$ & 1.451 & $\begin{array}{l}0.656- \\
3.211\end{array}$ & 0.358 & 1.188 & $\begin{array}{l}0.499- \\
2.826\end{array}$ & 0.70 \\
\hline & $\geq 70$ & 1 & & & 1 & & \\
\hline \multirow[t]{2}{*}{ mGPS } & 0 & 1 & & 0.144 & & & \\
\hline & $1+2$ & 0.545 & $\begin{array}{l}0.241- \\
1.230\end{array}$ & & & & \\
\hline \multirow[t]{2}{*}{ NLR } & $\geq 2.7$ & 0.231 & $\begin{array}{l}0.084- \\
0.631\end{array}$ & $0.004 * *$ & 0.275 & $\begin{array}{l}0.095- \\
0.796\end{array}$ & $0.017^{*}$ \\
\hline & $<2.7$ & 1 & & & 1 & & \\
\hline \multirow[t]{2}{*}{ PLR } & $\geq 1.55$ & 1.36 & $\begin{array}{l}0.569- \\
3.247\end{array}$ & 0.489 & & & \\
\hline & $<1.55$ & 1 & & & & & \\
\hline \multirow[t]{2}{*}{$\begin{array}{l}\text { CRP/Alb } \\
\text { ratio }\end{array}$} & $\geq 0.3$ & 0.358 & $\begin{array}{l}0.148- \\
0.867\end{array}$ & $0.023^{*}$ & 0.380 & $\begin{array}{l}0.135- \\
1.066\end{array}$ & 0.066 \\
\hline & $<0.3$ & 1 & & & 1 & & \\
\hline \multirow[t]{2}{*}{ PNI } & $>40$ & 0.605 & $\begin{array}{l}0.276- \\
1.328\end{array}$ & 0.211 & & & \\
\hline & $\leq 40$ & 1 & & & & & \\
\hline $\begin{array}{l}\text { mGPS, mo } \\
\text { lymphocyt }\end{array}$ & $\begin{array}{l}\text { d Glasgc } \\
\text { io; PNI, p }\end{array}$ & $\begin{array}{l}\text { Prognos } \\
\text { ognostic } r\end{array}$ & $\begin{array}{l}\text { core; NL } \\
\text { onal inc }\end{array}$ & utrophil-t & hocyte ra & PLR, pla & to- \\
\hline
\end{tabular}

\section{AEs}

The AEs during nal-IRI + 5-FU/I-LV treatment are summarized in Table 4. Adverse events were manageable, although gastrointestinal symptoms, such as nausea, loss of appetite, diarrhea and constipation, were observed. The most frequent grade $\geq 3 \mathrm{AE}$ was neutropenia, reported in $20 \%$ patients, followed by leukopenia, anemia, febrile neutropenia and loss of appetite. 
Table 4

Adverse events during nal-IRI + 5-FU/I-LV treatment.

\begin{tabular}{|lll|}
\hline Toxicity & All & Grade 3/4 \\
\hline Nausea & $12(27)$ & $1(2)$ \\
\hline Appetite loss & $15(34)$ & $2(5)$ \\
\hline Diarrhea & $10(23)$ & $0(0)$ \\
\hline Constipation & $13(30)$ & $0(0)$ \\
\hline Shingles & $1(2)$ & $0(0)$ \\
\hline Mucositis oral & $2(5)$ & $0(0)$ \\
\hline Malaise & $9(20)$ & $0(0)$ \\
\hline Ascites & $2(5)$ & $2(5)$ \\
\hline Dry skin & $1(2)$ & $0(0)$ \\
\hline Alopecia & $2(5)$ & $0(0)$ \\
\hline Dysgeusia & $3(7)$ & $0(0)$ \\
\hline Peripheral neuropathy & $13(30)$ & $0(0)$ \\
\hline Leukopenia & $15(34)$ & $4(9)$ \\
\hline Neutropenia & $15(34)$ & $9(20)$ \\
\hline Febrile neutropenia & $2(5)$ & $2(5)$ \\
\hline Thrombocytopenia & $4(9)$ & $0(0)$ \\
\hline Anemia & $8(18)$ & $3(7)$ \\
\hline AST increased & $4(9)$ & $1(2)$ \\
\hline ALT increased & $1(2)$ & $1(2)$ \\
\hline Hyponatremia & $1(2)$ & $1(2)$ \\
\hline Hypokalemia & $1(2)$ & $1(2)$ \\
\hline Creatinine increased & $1(2)$ & $0(0)$ \\
\hline
\end{tabular}

\section{Discussion}

The main finding of the present study was that nal-IRI + 5-FU/I-LV therapy was considered to be a useful regimen as second-line or later treatment for unresectable advanced pancreatic cancer, even in clinical practice. An NLR of $\geq 2.7$ was a significant predictive factor for the OS. 
In a systematic review of 71 studies in patients with unresectable advanced pancreatic cancer who received various second-line treatments, the median OS among all treatments ranged from 4.0 to 5.4 months [19]. Oxaliplatin has been investigated as a second-line treatment for patients with metastatic pancreatic cancer $[20,21]$. In the CONKO-3 trial, the median OS was 5.9 months for oxaliplatin + 5FU/folinic acid as second-line treatment after first-line gemcitabine monotherapy [20]. In the PANCREOX phase 3 study, the median OS was 6.1 months for biweekly modified FOLFOX6 as second-line treatment after first-line gemcitabine monotherapy [21]. In the present study, the median OS was 9.0 months, which was considered to be satisfactory in comparison with the previous regimen of second-line treatment.

The median OS and PFS were reported to be 6.2 (95\% Cl 4.8-8.4) months and 3.1 ( $95 \% \mathrm{Cl}$ 2.7-4.2) months, respectively, in the NAPOLI-1 study $[11,12]$. A phase II trial in Japan also demonstrated a similar median OS and PFS of 6.3 and 2.7 months, respectively, with a tolerable safety profile [13]. The median OS and PFS in the current study seemed to be slightly better than those in NAPOLI-1 study and the phase II trial in Japan. The ORR and DCR were previously reported to be $17 \%$ and $52 \%$, respectively, in the NAPOLI-1 study [11, 12], and a phase II trial in Japan demonstrated a similar ORR (17.5\%) and DCR (52.5\%) calculated based on the best response [13]. In the present study, the ORR and DCR were $5.3 \%$ and $44.7 \%$, respectively, which were slightly worse than those values in previous reports. The American Society of Clinical Oncology Clinical Practice Guidelines for the treatment of metastatic pancreatic cancer recommend nal-IRI + 5-FU/I-LV as second-line therapy in patients previously treated with gemcitabine plus nab-paclitaxel [22], and the current National Comprehensive Cancer Network guidelines for the treatment of pancreatic cancer recommend nal-IRI + 5-FU/I-LV as category 1 second-line therapy for metastatic disease [23].

The NLR showed a significant difference as a predictive factor for the OS and response in the present study. Inflammation has recently been considered to play an essential role in cancer progression. A number of inflammation-based prognostic factors have been developed, including the GPS, mGPS, PLR, NLR, CRP/Alb ratio and PNI [16-18, 24-26]. Iwai et al. [27]. reported that a high NLR might be an independent indicator of a poor prognosis in patients with unresectable pancreatic cancer. In their study, the NLR was the best predictive factor among the GPS, mGPS, PLR, CRP/Alb ratio and PNI [27]. Although all of these inflammation-based prognostic factors reached statistical significance in their study [27], only the NLR and CRP/Alb ratio reached statistical significance in the present study. In their receiver operator characteristic analyses for the OS at six-month follow-up, the AUC area was the greatest for the NLR, followed in descending order by the CRP/Alb ratio, GPS, PNI, MGPS and PLR [27]. The sequence of the AUC area in the present study was similar to the previous report: NLR, CRP/Alb ratio, mGPS, PNI and PLR. If the number of patients were increased in our study, not only the NLR and CRP/Alb ratio but also other factors, namely the mGPS, PNI and PLR, might have also shown statistical significance.

The mechanism underlying the relationship between the NLR and prognosis in patients with unresectable pancreatic cancer remains to be clarified. Neutrophils inhibit the immune response by lymphocytes, natural killer cells or activated T cells $[28,29]$, while lymphocytes reflect the immune response of the host to either infection or cancer. Tumor-infiltrating lymphocytes are reported to be associated with a good 
prognosis in patients with pancreatic ductal adenocarcinoma [30]. Baseline characteristics associated with long-term survivors who survived for more than one year in the NAPOLI-1 study $[11,12]$ were a younger age, better performance status, lower NLR, lower CA19-9 level and absence of liver metastases. Six of the 44 patients survived for more than one year from start of nal-IRI + 5-FU/LV treatment in the present study. Although the number of long-term survivors in our study was small, a lower NLR and lower CA19-9 level seemed to be associated with a long-term survival.

AEs were manageable, although gastrointestinal symptoms and blood cell AEs were observed. The most common grade $\geq 3 \mathrm{AE}$ in this study was neutropenia (20.0\%), followed by leukopenia (9.1\%) and anemia (6.1\%). In the NAPOLI-1 trial, grade $\geq 3$ AEs included neutropenia (15.4\%), a decreased white blood cell count $(12.0 \%)$ and diarrhea $(9.4 \%)[11,12]$.

The current study had several limitations. First, this was a retrospective study, and the number of patients was relatively small. Because the number of patients with prior irinotecan-based therapy was small, the effect of a history of irinotecan-based therapy could not be analyzed. Future studies should explore this point.

In conclusion, nal-IRI + 5-FU/I-LV therapy was considered to be a useful regimen as second-line or later treatment for unresectable advanced pancreatic cancer, even in clinical practice. An NLR of $\geq 2.7$ was a significant predictive factor. Nal-IRI + 5-FU/I-LV therapy showed a good response with manageable AEs.

\section{Declarations}

\section{Conflict of Interest: None}

Declarations: This study protocol was approved by the ethics committee of each institution and was conducted in compliance with the 1975 Declaration of Helsinki.

\section{References}

1. Siegel RL, Miller KD, Jemal A. Cancer statistics, 2020. CA Cancer J Clin. 2020;70:7-30.

2. Ferlay J, Ervik M, Lam F, et al. Global cancer observatory: cancer today. Lyon: International Agency for Research on Cancer; 2018. https://gco.iarc.fr/today.

3. Frampton JE. Liposomal Irinotecan: A Review in Metastatic Pancreatic Adenocarcinoma. Drugs. 2020;80:1007-18.

4. Orth $M$, Metzger P, Gerum S, et al. Pancreatic ductal adenocarcinoma: biological hallmarks, current status, and future perspectives of combined modality treatment approaches. Radiat Oncol. 2019;14:141.

5. Uccello M, Moschetta M, Mak G, et al. Towards an optimal treatment algorithm for metastatic pancreatic ductal adenocarcinoma (PDA). Curr Oncol. 2018;25:e90-4. 
6. Von Hoff DD, Ervin T, Arena FP, et al. Increased survival in pancreatic cancer with nab-paclitaxel plus gemcitabine. N Engl J Med. 2013;369:1691-703.

7. Conroy $T$, Desseigne $F$, Ychou $M$, et al. FOLFIRINOX versus gemcitabine for metastatic pancreatic cancer. N Engl J Med. 2011;364:1817-25.

8. Burris HA III, Moore MJ, Andersen J, et al. Improvements in survival and clinical benefit with gemcitabine as firstline therapy for patients with advanced pancreas cancer: a randomized trial. $J$ Clin Oncol. 1997;15:2403-13.

9. Chiang NJ, Chao TY, Hsieh RK, et al. A phase I dose-escalation study of PEP02 (irinotecan liposome injection) in combination with 5-fluorouracil and leucovorin in advanced solid tumors. BMC Cancer. 2016;16:907.

10. Drummond DC, Noble CO, Guo Z, et al. Development of a highly active nanoliposomal irinotecan using a novel intraliposomal stabilization strategy. Cancer Res. 2006;66:3271-7.

11. Wang-Gillam A, Li CP, Bodoky G, Dean A, et al. Nanoliposomal irinotecan with fluorouracil and folinic acid in metastatic pancreatic cancer after previous gemcitabine-based therapy (NAPOLI-1): a global, randomised, open-label, phase 3 trial. Lancet. 2016;387:545-57.

12. Wang-Gillam A, Hubner RA, Siveke JT, et al. NAPOLI-1 phase 3 study of liposomal irinotecan in metastatic pancreatic cancer: final overall survival analysis and characteristics of long-term survivors. Eur J Cancer. 2019;108:78-87.

13. Ueno M, Nakamori S, Sugimori K, et al. nal-IRI+5-FU/LV versus 5-FU/LV in post-gemcitabine metastatic pancreatic cancer: Randomized phase 2 trial in Japanese patients. Cancer Med. 2020;9:9396-408.

14. Oken MM, Creech RH, Tormey DC, et al. Toxicity and response criteria of the Eastern Cooperative Oncology Group. Am J Clin Oncol. 1982;5:649-55.

15. NCl Common Terminology Criteria for Adverse Events (CTCAE). ver 5.0. https://ctep.cancer.gov/protocolDevelopment/electronic_applications/ctc.htm Accessed March 1, 2021.

16. McMillian DC, Crozier JE, Canna K, et al. Evaluation of an inflammation-based prognostic score (GPS) in patients undergoing resection for colon and rectal cancer. Int $\mathrm{J}$ Colorectal Dis. 2007;22:881-6.

17. Inoue $Y$, Iwata $T$, Okugawa $Y$, et al. Prognostic significance of a systemic inflammatory response in patients undergoing multimodality therapy for advanced colorectal cancer. Oncology. 2013;84:1007.

18. Walsh SR, Cook EJ, Goulder F, et al. Neutrophil-lymphocyte ratio as a prognostic factor in colorectal cancer. J Surg Oncol. 2005;91:181-4.

19. Nagrial AM, Chin VT, Sjoquist KM, et al. Second-line treatment in inoperable pancreatic adenocarcinoma: a systematic review and synthesis of all clinical trials. Crit Rev Oncol Hematol. 2015;96:483e97. 
20. Oettle $\mathrm{H}$, Riess $\mathrm{H}$, Stieler JM, et al. Second-line oxaliplatin, folinic acid, and fluorouracil versus folinic acid and fluorouracil alone for gemcitabine-refractory pancreatic cancer: outcomes from the CONKO003 trial. J Clin Oncol. 2014;32:2423e9.

21. Gill S, Ko YJ, Cripps C, et al. PANCREOX: a randomized phase III study of 5-fluorouracil/leucovorin with or without oxaliplatin for secondline advanced pancreatic cancer in patients who have received gemcitabine-based chemotherapy. J Clin Oncol. 2016;4:3914e20.

22. Sohal DPS, Kennedy EB, Khorana A, et al. Metastatic pancreatic cancer: ASCO clinical practice guideline update. J Clin Oncol. 2018;36:2545e56.

23. NCCN Clinical Practice Guidelines in Oncology. Pancreatic adenocarcinoma version 1. 2019. National Comprehensive Cancer Network; 2018.

24. Network; 2018. Smith RA, Bosonnet L, Raraty M, et al. Preoperative platelet-lymphocyte ratio is an independent significant prognostic marker in resected pancreatic ductal adenocarcinoma. Am J Surg. 2009;197:466-72.

25. Haruki K, Shiba H, Shirai Y, et al. The C-reactive Protein to Albumin Ratio Predicts Long-Term Outcomes in Patients with Pancreatic Cancer After Pancreatic Resection. World J Surg. 2016;40:2254-60.

26. Ikeguchi M, Hanaki T, Endo K, et al. C-Reactive Protein/Albumin Ratio and Prognostic Nutritional Index Are Strong Prognostic Indicators of Survival in Resected Pancreatic Ductal Adenocarcinoma. J Pancreat Cancer. 2017;3:31-6.

27. Iwai $\mathrm{N}$, Okuda T, Sakagami J, et al. Neutrophil to lymphocyte ratio predicts prognosis in unresectable pancreatic cancer. Sci Rep. 2020;10:18758.

28. Petrie HT, Klassen LW, Kay HD. Inhibition of human cytotoxic T lymphocyte activity in vitro by autologous peripheral blood granulocytes. J Immunol. 1985;134:230-4.

29. el-Hag A, Clark RA. Immunosuppression by activated human neutrophils. Dependence on the myeloperoxidase system. J Immunol. 1987;139:2406-13.

30. Lianyuan T, Dianrong $X$, Chunhui $Y$, et al. The predictive value and role of stromal tumor-infiltrating lymphocytes in pancreatic ductal adenocarcinoma (PDAC). Cancer Biol Ther. 2018;19:296-305.

\section{Figures}




\section{Figure 1}

(a)

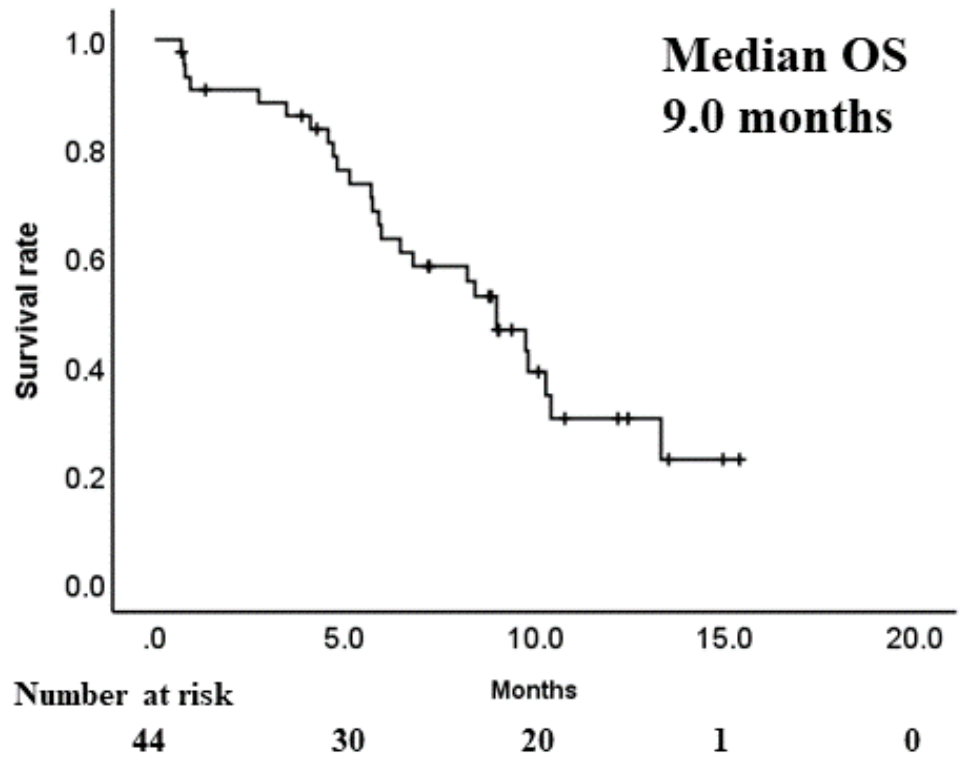

(b)

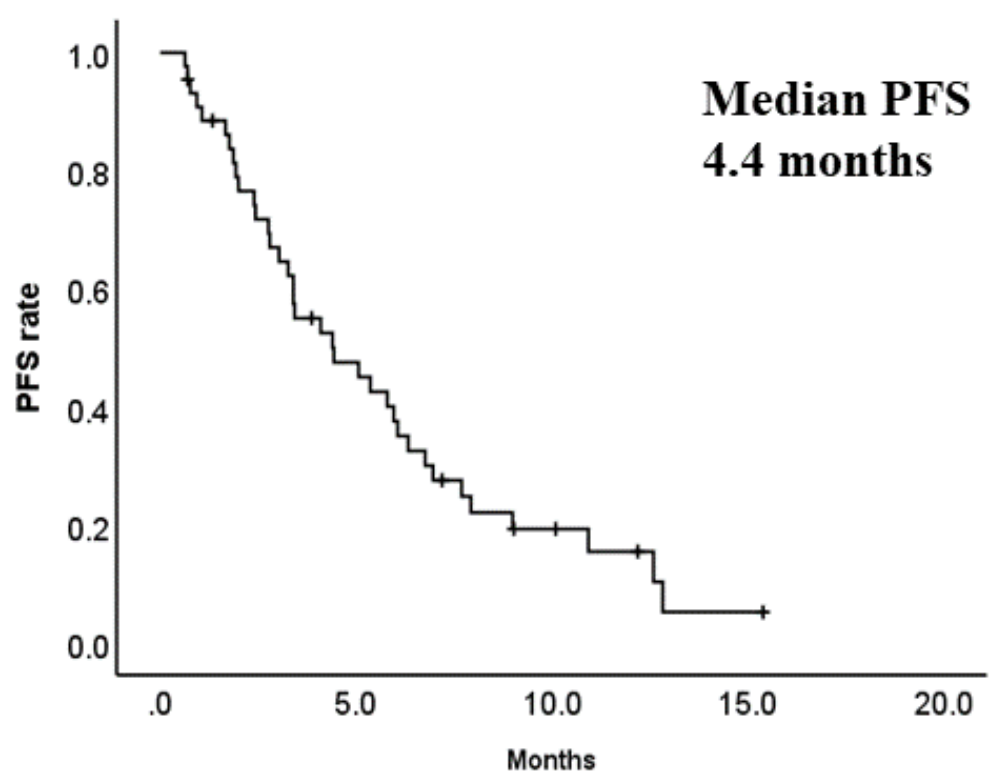

Number at risk

$\begin{array}{lllll}44 & 19 & 6 & 1 & 0\end{array}$

Figure 1

(a) The overall survival (OS) with nano-liposomal irinotecan plus 5-fluorouracil/-leucovorin (nal-IRI + 5FU/I-LV). The Kaplan-Meier curve showed that the median OS of all patients was 9.0 months. (b) The progression-free survival (PFS) with nal-IRI + 5-FU/I-LV. The median PFS in all patients was estimated to be 4.4 months. 
Figure 2 (a)

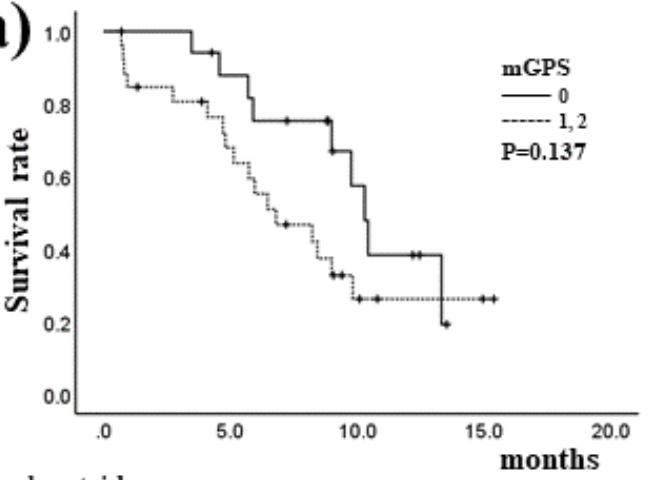

Number atrisk mGPS $0 \quad 18$ mGPS 1,2 26

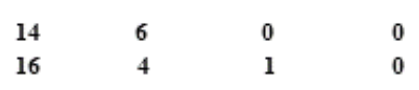

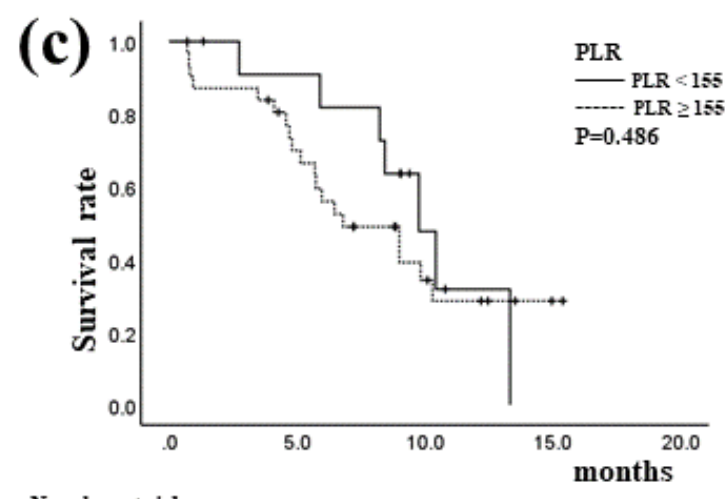
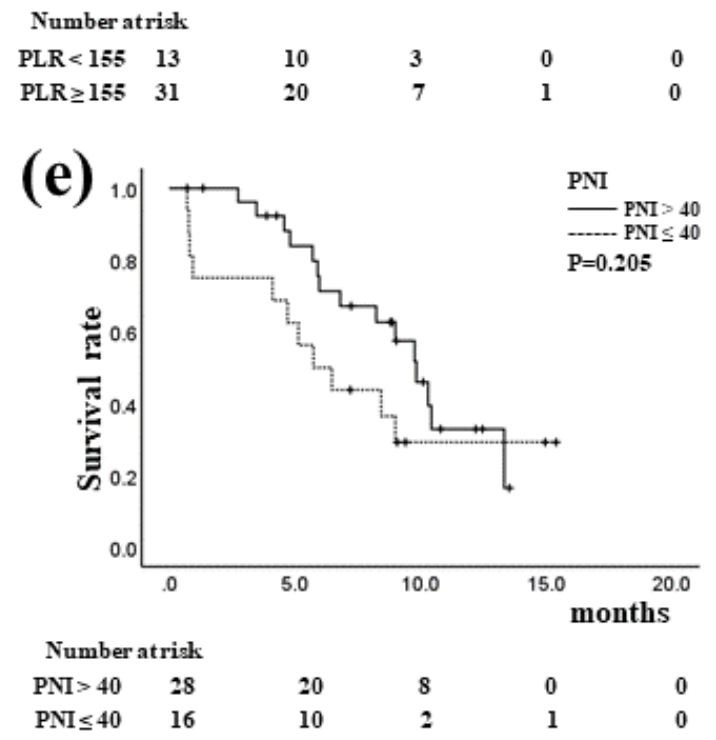
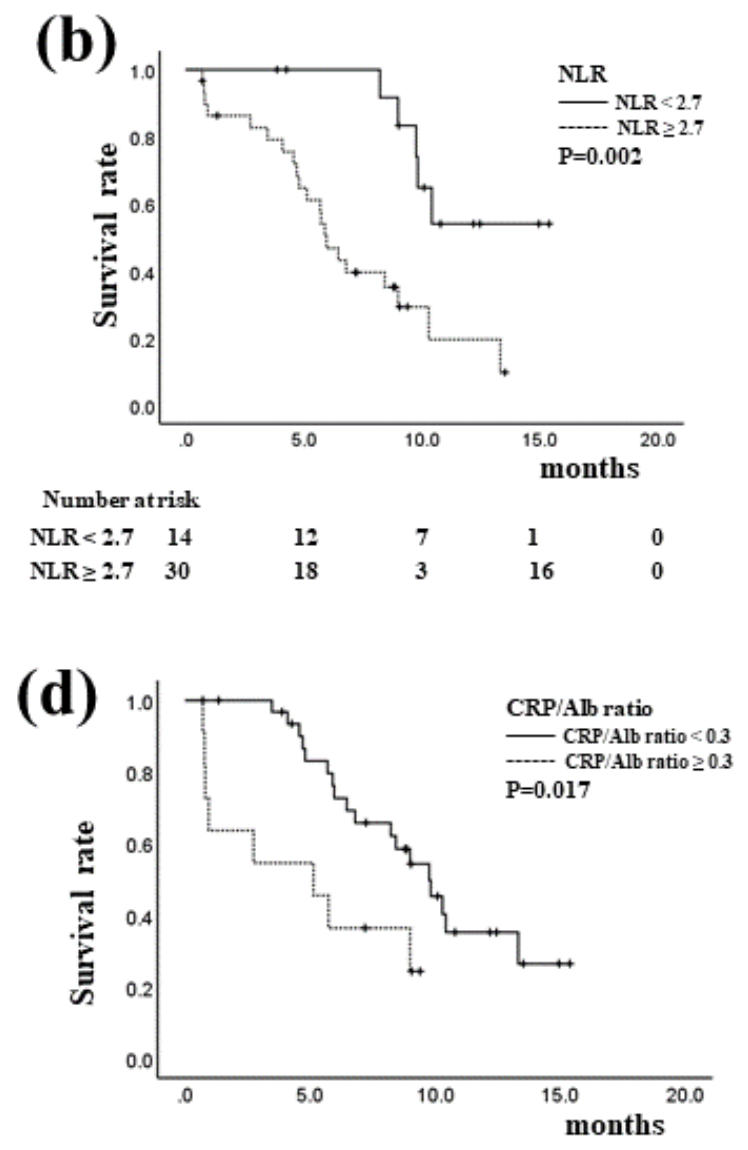

Number atrisk
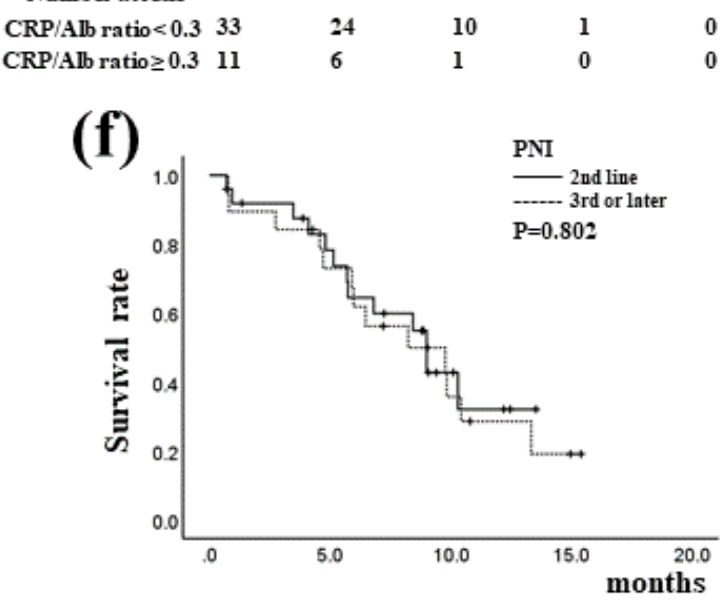

Number atrisk 2nd line 25 3rd or later 19

\section{Figure 2}

The Kaplan-Meier curve according to the systemic inflammation-based prognostic indicators and lines of treatment. (a) The modified Glasgow Prognostic Score (mGPS). There were no significant differences between the patients with an mGPS score 0 (median OS, 10.3 months; $95 \% \mathrm{Cl}, 9.31-11.29$ ) and those with an mGPS score $1+2$ (median OS, 6.8 months; $95 \%$ Cl, 3.36-10.24; $\mathrm{P}=0.137$ ) (b) The neutrophil-tolymphocyte ratio (NLR). The patients with an NLR $<2.7$ had a significantly better survival (median OS, not 
reached) than those with an NLR $\geq 2.7$ (median OS, 6.0 months; 95\% $\mathrm{Cl}, 4.98-6.95 ; \mathrm{P}=0.002$ ). (c) The platelet-to-lymphocyte ratio (PLR). There were no significant differences between the patients with a PLR $<155$ (median OS, 9.8 months; 95\% Cl, 7.60-11.93) and those with a PLR $\geq 155$ (median OS, 6.8 months; $95 \% \mathrm{Cl}, 3.52-10.08 ; \mathrm{P}=0.486$ ). (d) CRP/Alb ratio. The patients with a CRP/Alb ratio $<0.3$ also had a significantly better survival (median OS, 9.8 months; $95 \% \mathrm{Cl}, 7.82-11.71$ ) than those with a CRP/Alb ratio $\geq 0.3$ (median OS, 5.1 months, $95 \% \mathrm{Cl}, 0.0-10.31 ; \mathrm{P}=0.017$ ). (e) The prognostic nutritional index (PNI). There were no significant differences between the patients with a PNI > 40 (median OS, 9.8 months; $95 \%$ $\mathrm{Cl}, 8.28-11.39$ ) and those with a PNI $\leq 40$ (median OS, 5.7 months; $95 \% \mathrm{Cl}, 3.12-8.35 ; \mathrm{P}=0.205$ ). (f) Line of treatment. There were no significant differences between the patients who received nal-IRI + 5-FU/I-LV as 2nd-line treatment (median 9.0 months; $95 \% \mathrm{Cl}$ 6.2-11.8 months) and those treated with nal-IRI + 5FU/I-LV as 3rd-line or later treatment (median 8.2 months; $95 \% \mathrm{Cl}$ 2.5-14.0 months; $\mathrm{P}=0.802$ ). 


\section{Figure 3}

(a)

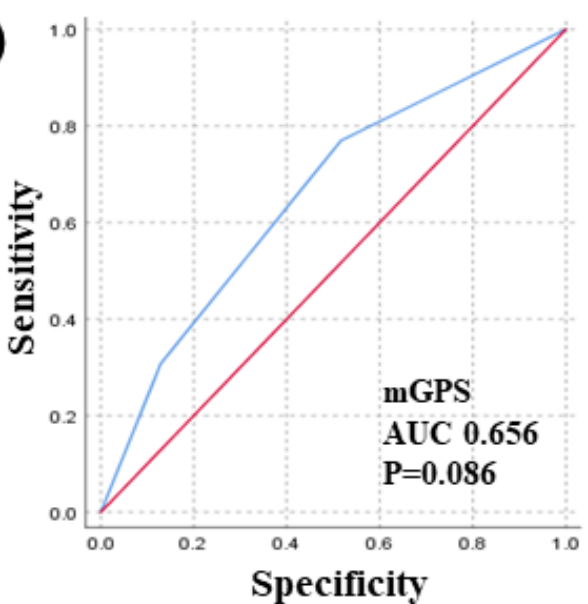

(c)

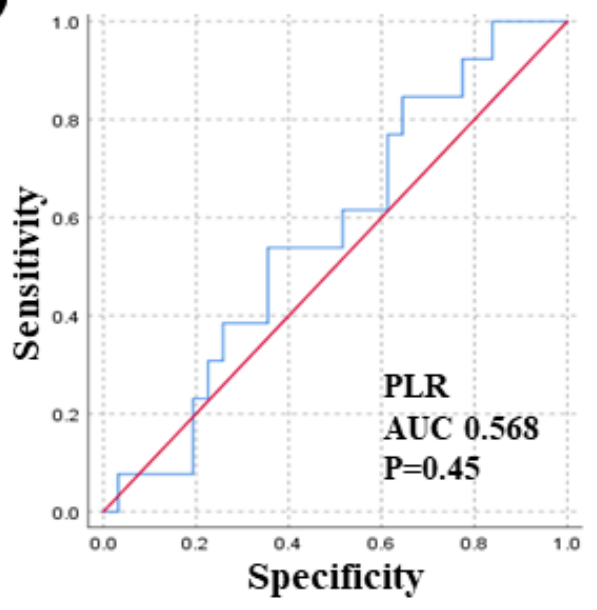

(e)

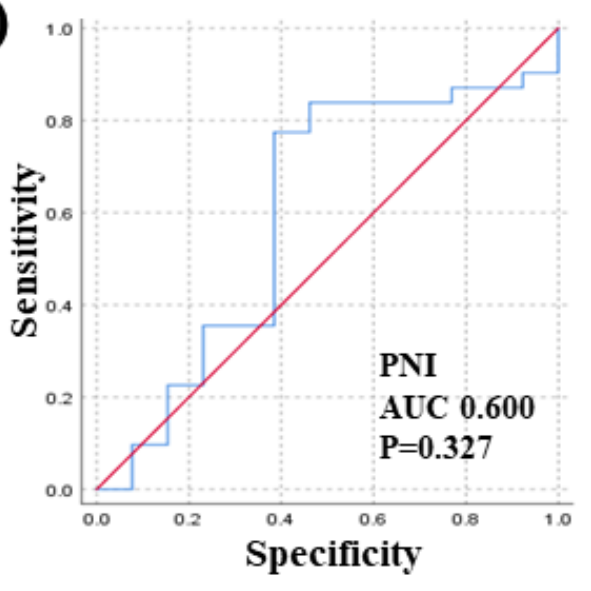

(b)

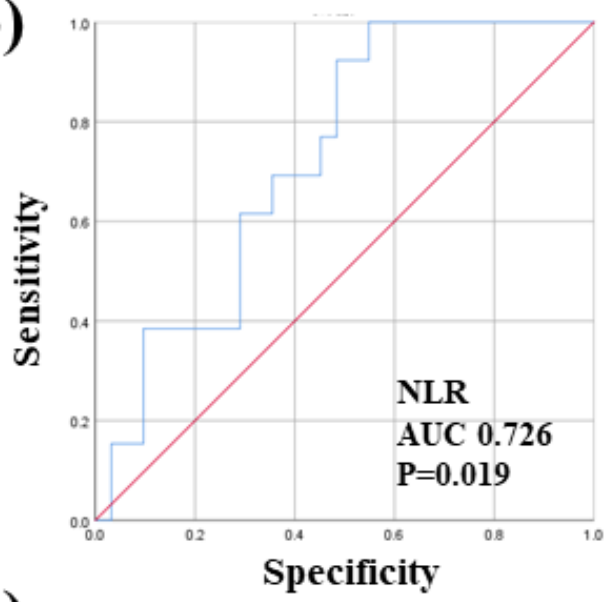

(d)

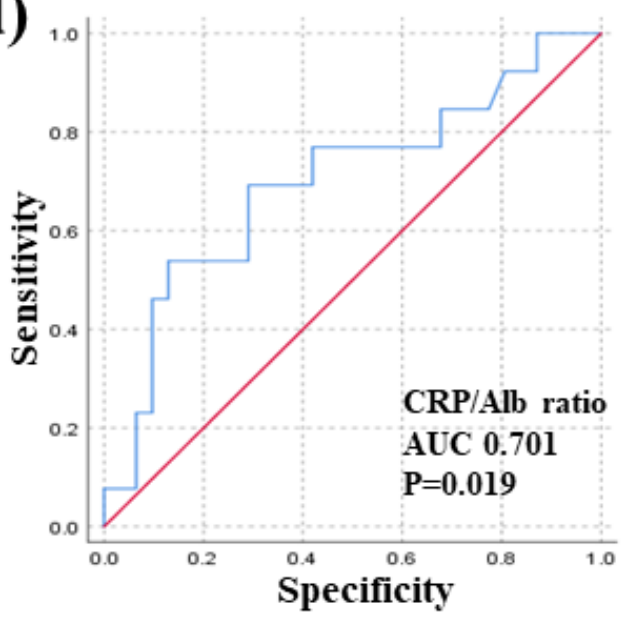

\section{Figure 3}

Receiver operator characteristic (ROC) analyses for the overall survival (OS) at 6-month follow-up. The (a) modified Glasgow Prognostic Score (mGPS), (b) neutrophil-to-lymphocyte ratio (NLR), (c) platelet-tolymphocyte ratio (PLR), (d) CRP/Alb ratio and (e) prognostic nutritional index (PNI). The area under the curve (AUC) of variables was as follows: mGPS, 0.656 (95\% Cl 0.478-0.835, $\mathrm{P}=0.086)$ : NLR, 0.726 (95\% Cl 0.578-0.876, $\mathrm{P}=0.019)$ : PLR, 0.568 (95\% Cl 0.391-0.745, $\mathrm{P}=0.45)$ : CRP/Alb ratio, 0.701 (95\% Cl 0.521- 
0.881, $\mathrm{P}=0.028)$ : $\mathrm{PNI}, 0.600$ (95\% $\mathrm{Cl} 0.399-0.802, \mathrm{P}=0.327)$. The NLR and CRP/Alb ratio were significantly associated with the OS at 6-month follow-up, while the MGPS, PLR and PNI did not influence the OS at 6month follow-up. 